\title{
Assessment on Structural Integrity of In-service Machine Using De-noised Vibrational Modal Data and Artificial Neural Network
}

\author{
Zhi Chao Ong ${ }^{1, a}$, Ee Teng Yap ${ }^{1}$, Zubaidah Ismail ${ }^{2}$ and Shin Yee Khoo ${ }^{1}$ \\ ${ }^{1}$ Department of Mechanical Engineering, Faculty of Engineering, University of Malaya, 50603 Kuala Lumpur, \\ Malaysia \\ ${ }^{2}$ Department of Civil Engineering, Faculty of Engineering, University of Malaya, 50603 Kuala Lumpur, Malaysia
}

\begin{abstract}
The recent oil price drop creates a demand for swift action within oil and gas industry to shift focus from increasing daily production rates, to optimizing existing assets in achieving growth. Industrial machinery, one of the industry's key asset many times failed due to high amplitude vibration that contributes to accelerated wear and tear and subsequently results in high cycle fatigue failure. As such there is a need to develop a structural integrity assessment for in-service machinery for continuous and safe operation. Vibration-based method such as Experimental Modal Analysis (EMA) is widely used for damage detection on civil and piping system under stationary environment. However, in industrial applications, system shutdown is very costly. EMA is also undesirable in this case due to the dominant ambient and system disturbances on the in-service system. An alternative method called Impact-Synchronous Modal Analysis (ISMA) is developed to perform modal analysis under noisy environment. Applying the ISMA technique in de-noising the non-synchronous disturbances at upstream could generate a cleaner and static-like modal data downstream for analysis. Artificial Neuron Networks (ANN) is then applied extensively in structural damage identification purposes based on changes in modal data due to its excellent pattern recognition ability. By leveraging on the latest technologies, i.e. ISMA and ANN as proposed, it allows real-time monitoring of assets, in this case, the machines, as well as the ability to transform continuous streams of data into useful information to predict damages.
\end{abstract}

\section{Introduction}

The recent drop in oil price creates a demand for swift action to shift focus from increasing daily production rates to optimizing existing assets in achieving growth. Development of a structural integrity assessment could allow continuous monitoring the health of the system and notify the enduser prior to any failure and to promote high health, safety, environment and quality (HSEQ) and cost efficiency to achieve the organizations operational and financial objectives.

Detection of damage is one of the most important factors in maintaining the integrity and safety of structures. Vibration-based methods are a global and non-destructive damage identification approach

\footnotetext{
${ }^{\mathrm{a}}$ Corresponding author : alexongzc@um.edu.my
} 
that has been considered for use on the dynamic properties of structure to identify the location and severity of damage without prior information of the type or level of damage. Many researchers focused on damage detection of civil structures and piping system where clean vibrational modal data could be obtained using the conventional Experimental Modal Analysis (EMA) under stationary environment [1-5]. However, the suitability of the modal parameters in damage detection is heavily affected by the ambient conditions and the consistency and reliability of the testing procedures. Downstream modal parameters could be contaminated by measurement error as well as modal extraction error. In addition, in industrial applications, shutting down the system could induce high downtime cost to the organizations. EMA is also undesirable in cases where it is to be performed on the in-service systems where the ambient and system disturbances dominate. It will be an uphill challenge when the same algorithms are applied in a noisy environment of in-service systems.

Most of vibration-based damage identification techniques are considered as some form of pattern recognition since they look for differences between two categories, e.g. before and after a structure is damaged or differences in damage levels. Artificial Neural Networks (ANNs) have been applied extensively in recent years due to their excellent pattern recognition ability, which is useful for structural damage identification purposes based on the change in modal analysis data [6-8]. Previous researches show that ANN and pattern recognition techniques have been mostly applied on stationary civil structures, beam-like and pipe-like structures damage detection. However, the nature of noisy ambient and system disturbances from in-service systems remain the main challenge for the researchers because it produces noisy modal data for damage detection or structural integrity assessment.

An alternative modal analysis method known as Impact-Synchronous Modal Analysis (ISMA) is developed which allows modal analysis to be performed in a noisy environment, when structure is in service and yet could provide a static-like vibrational modal data through de-noising processes $[9,10]$. ISMA is considered a more practical technique to adopt in obtaining the modal data for the use of damage detection. Therefore, this study investigates a different form of parameters at the upstream of modal data, i.e. real time de-noised frequency response vectors combined with ANN with the aim to develop a damage detection scheme under noisy environment in a more practical way. Applying the ISMA technique which is a practical approach in de-noising the non-synchronous disturbances at the upstream could generate a cleaner and static-like modal data at downstream for a more accurate damage detection scheme under ANN environment.

\section{Methods}

\subsection{Experimental Set-up}

A rectangular Perspex plate with a dimension of $48 \mathrm{~cm} \times 20 \mathrm{~cm} \times 0.9 \mathrm{~cm}$ (width $\mathrm{x}$ height $\mathrm{x}$ thickness), weighting $1.1 \mathrm{~kg}$, was used as the test specimen as shown in Figure 1 to simulate a similar vibration behaviour of an automobile. The plate was ground supported using screws at each of the four corners. It was connected to the steel plate and aluminium supports at every corners. The ground supports acted as the suspension/spring components of a typical automobile wheels.

The plate was divided into nine (9) measurement points. Since the plate is treated as an automobile, no sensor can be mounted on the wheel (located at the edges of the plate; point 1, 3, 7 and 9). By mounting it on the body it is still possible to find the damages or loss of stiffness at the wheel or suspension. Therefore, five (5) accelerometers were mounted in symmetric order on the plate as shown in Figure 2. The accelerometers used were Wilcoxon Research model S100C accelerometer which has a built-in charge amplifier. The mounting method used on the accelerometer is by cyanoacrylate adhesive. A manual PCB model 086C03 impact hammer was used to create an impact at the edges of plate structure / ground support for analysing its dynamic behaviour due to impact excited vertically from the automobile wheels. As for the DAQ hardware system, National Instrument-Universal Serial Bus (NI-USB 9234) signal acquisition module was used in this setup to acquire six (6) dynamic signals (1 impact hammer as input signal and 6 accelerometers as output 
signals). The DAQ hardware system will send the acquired data to the computer software, LabVIEW 2013 for the post-processing to acquire the FRF. Modal analysis was performed in two conditions, i.e. stationary and in-service conditions using EMA and ISMA methods. To create the in-service condition, an electrodynamic shaker (LDS model V201) with external power amplifier (LDS model PA25E) was used to produce a harmonic excitation at $30 \mathrm{~Hz}$.

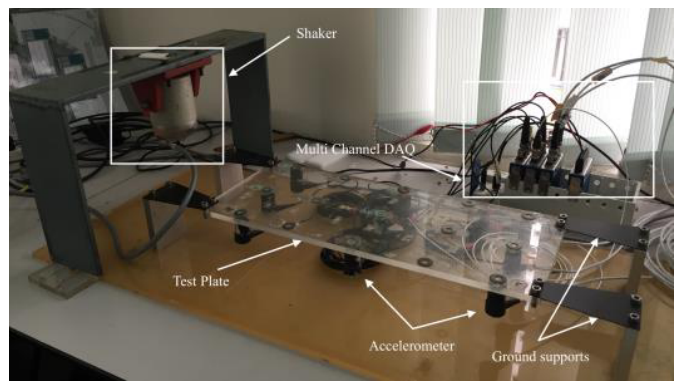

Figure 1. Experimental set-up of test rig

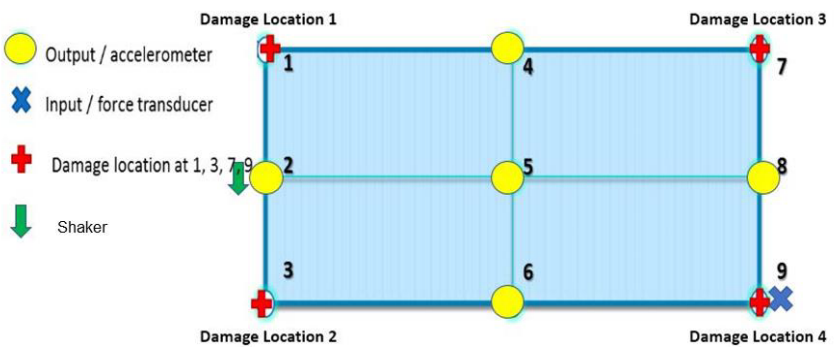

Figure 2. Sensor and damage location

\subsection{Damage Identification Scheme}

Since the plate simulate a four-wheel car, the location of the damage usually happens at the suspension part where it absorbs the impact done by the tire when hitting a pothole or rough road. To simulate a damage condition, the screws that hold the plate and ground supports together were used as the damage point. The screws will be removed and loosen to an extent of shift in natural peaks were observed to simulate the damage conditions under different severities. Table 1 shows the list of condition along with its description.

Table 1. Damage conditions and descriptions.

\begin{tabular}{|l|l|}
\hline \multicolumn{1}{|c|}{ Conditions } & \multicolumn{1}{c|}{ Description } \\
\hline Undamaged (UD) & All four (4) points of the screws were tighten \\
\hline High Severity Damaged Location 1 (HD-1) & Screws at point 1 were removed, as for point 3, 7, and 9 were tighten \\
\hline High Severity Damaged Location 2 (HD-2) & Screws at point 3 were removed, as for point 1, 7, and 9 were tighten \\
\hline High Severity Damaged Location 3 (HD-3) & Screws at point 7 were removed, as for point 1, 3, and 9 were tighten \\
\hline High Severity Damaged Location 4 (HD-4) & Screws at point 9 were removed, as for point 1, 3, and 7 were tighten \\
\hline Low Severity Damaged Location 1 (LD-1) & Screws at point 1 were loosen, as for point 3, 7, and 9 were tighten \\
\hline Low Severity Damaged Location 2 (LD-2) & Screws at point 3 were loosen, as for point 1, 7, and 9 were tighten \\
\hline Low Severity Damaged Location 3 (LD-3) & Screws at point 7 were loosen, as for point 1, 3, and 9 were tighten \\
\hline Low Severity Damaged Location 4 (LD-4) & Screws at point 9 were loosen, as for point 1, 3, and 7 were tighten \\
\hline
\end{tabular}


Neural network pattern recognition toolbox in MATLAB was used in developing the damage identification scheme in this study. The toolbox solves pattern-recognition classification problems using a two-layer feed-forward pattern recognition neural network. It was trained to classify inputs according to the target classes. Total of 45 sets of FRF data at the frequency range $0 \mathrm{~Hz}$ to $199.5 \mathrm{~Hz}(9$ damage conditions x 5 sensor points) were used and input into pattern recognition neural network for training. The size of input is $400(0.5 \mathrm{~Hz}$ frequency resolution gives $2 \times 200$ input data). Each set of data was trained with their target data of 1 at respective row to differentiate the condition and detect damage. The setting was set by dividing the input data into $90 \%$ of training data, $5 \%$ of validation data and $5 \%$ of testing data with the number of hidden neurons of 400 . The data with confusion matrix approaching $100 \%$ indicates the data were well-trained. Any set of untrained data can be input into the trained network to identify the condition in the system.

\section{Results and Discussions}

\subsection{Frequency Response Functions Analysis}

From the FRF graph analysis, it is found that the peaks at each FRF for a specific condition is distinct and carrying its own dynamic behaviour. When the structure is damaged, the peaks at FRFs graph are shifted to the left side towards lower frequency range due to the loss of stiffness of the system. In addition, these FRFs obtained for a specific damage condition at their respective sensor point consistent and stable during the real time measurement This shows that directly-measured FRF is easy to obtain, reliable and sensitive to be used as input for damage detection process. In ISMA, ISTA averaging technique is utilized to de-noise the unaccounted forces exerted by the shaker. This can be seen from Figure 3 where no much differences between FRFs graph pattern of EMA and ISMA at the same sensor point under same damage condition is observed. Both the frequency and magnitude at which modes exist in both EMA and ISMA show very high similarity. Hence, it is proven that ISMA is reliable by providing static comparable FRF data through its de-noising method during in-service condition.
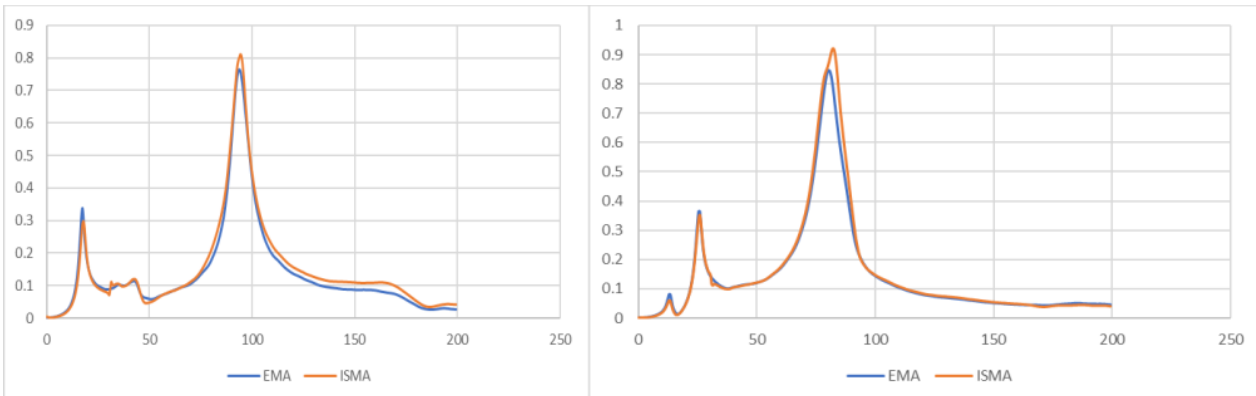

Figure 3. Comparison of EMA and ISMA FRFs for (a) UD condition, (b) HD-1 condition at Point 5

\subsection{ANN Model Validation}

The output data after training is Tabulated in Table 2, it is obvious that all the data are successfully trained with accuracy approaching $100 \%$. It is observed that only sensor Point 2 and Point 8 which are slightly deviated from the target data of ' 1 ' with very small standard deviations of 0.001154 and 0.000767 respectively. Hence, this training network is considered a good damage detection scheme. 
Table 2. Output data after training.

\begin{tabular}{|l|l|l|l|l|l|l|l|l|l|l|}
\hline $\begin{array}{l}\text { Condition } \\
\text { Point }\end{array}$ & UD & HD-1 & HD-2 & HD-3 & HD-4 & LD-1 & LD-2 & LD-3 & LD-4 & $\begin{array}{l}\text { Standard } \\
\text { Deviation }\end{array}$ \\
\hline Point 2 & 0.9966 & 1.0000 & 1.0000 & 0.9988 & 1.0000 & 1.0000 & 1.0000 & 1.0000 & 1.0000 & 0.001154 \\
\hline Point 4 & 1.0000 & 1.0000 & 1.0000 & 1.0000 & 1.0000 & 1.0000 & 1.0000 & 1.0000 & 1.0000 & 0 \\
\hline Point 5 & 1.0000 & 1.0000 & 1.0000 & 1.0000 & 1.0000 & 1.0000 & 1.0000 & 1.0000 & 1.0000 & 0 \\
\hline Point 6 & 1.0000 & 1.0000 & 1.0000 & 1.0000 & 1.0000 & 1.0000 & 1.0000 & 1.0000 & 1.0000 & 0 \\
\hline Point 8 & 1.0000 & 1.0000 & 1.0000 & 1.0000 & 0.9977 & 1.0000 & 1.0000 & 1.0000 & 1.0000 & 0.000767 \\
\hline
\end{tabular}

It is noted that all the conditions are successfully classified into their respective damage locations and severities for all sensor points with the maximum deviation of only 0.0034 (1.0000-0.9966) which is very approaching the target data (1.0000). Data of sensor Point 4, Point 5 and Point 6 are 100\% similar with target data with no deviation. This show the consistency of the FRF data obtained and its suitability to be used as input in damage detection scheme. The damage detection scheme is validated to be successful under stationary condition in which sensor Point 4, Point 5 and Point 6 are recommended to be used for testing and actual implementation of the scheme.

Following this, the damage detection scheme is used to test and validate with other sets of untrained data. ISMA FRF data which collected under in-service condition is input into the damage detection scheme and the testing result is shown in Table 3.

Table 3. Testing of untrained ISMA data.

\begin{tabular}{|l|c|c|c|c|c|c|c|c|c|c|}
\hline $\begin{array}{l}\text { Condition } \\
\text { Point }\end{array}$ & UD & HD-1 & HD-2 & HD-3 & HD-4 & LD-1 & LD-2 & LD-3 & LD-4 & $\begin{array}{l}\text { Standard } \\
\text { Deviation }\end{array}$ \\
\hline Point 2 & 0.9883 & 1.0000 & 0.9984 & 0.8430 & 0.9999 & 0.9969 & 1.0000 & 1.0000 & 0.9999 & 0.051780 \\
\hline Point 4 & 0.9997 & 1.0000 & 1.0000 & 0.9071 & 1.0000 & 1.0000 & 0.9969 & 1.0000 & 1.0000 & 0.030842 \\
\hline Point 5 & 0.9999 & 1.0000 & 1.0000 & 0.9999 & 0.9996 & 1.0000 & 1.0000 & 0.9998 & 1.0000 & 0.000136 \\
\hline Point 6 & 1.0000 & 1.0000 & 1.0000 & 0.9926 & 1.0000 & 0.9998 & 1.0000 & 0.9988 & 1.0000 & 0.002440 \\
\hline Point 8 & 0.9998 & 0.9999 & 0.9998 & 0.9967 & 0.9990 & 1.0000 & 0.9980 & 1.0000 & 1.0000 & 0.001164 \\
\hline
\end{tabular}

It is noted that for HD-3 at Point 2, the deviation is more than $10 \%$ in which $0.8430(84.30 \%)$ indicates high level of severity at damage location 3 while $0.1535(15.35 \%)$ indicates low level of severity at the same damage location 3 . This is due to the non-significance difference in the collected FRF data for these 2 conditions (HD-3 and LD-3) as shown in Figure 4. It is shown that the first peak of FRFs for high severity (HD-3) has minimal down shift in frequency compared with the low severity case (LD-3) whereas the rest of the peaks remain unchanged for both cases. This indicates the effect of damage severity at location 3 on the change of FRF at sensor Point 2 is minimal. It is also noted that the largest standard deviations of result data happened at Point 2 with 0.051780 . Therefore, sensor Point 2 is not recommended to be used for testing or actual implementation of the scheme.

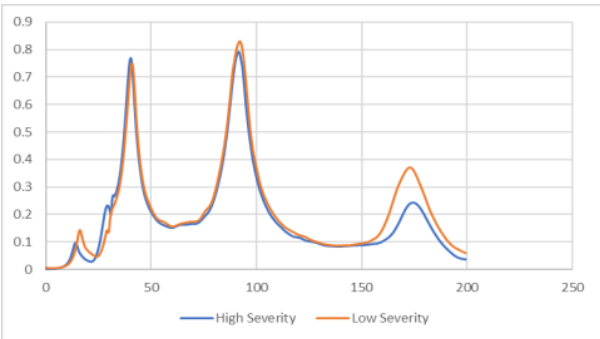

Figure 4. Comparison of FRFs of HD-3 and LD-3 condition at Point 2

Among all five sensor points used, it is observed that sensor Point 5 is the point with smallest standard deviation which is 0.000136 . Hence, sensor Point 5 is considered as the best sensor point to be used for testing and actual implementation of the scheme under in-service condition. The 
developed damage detection scheme proves that the damage detection scheme is successfully developed with $100 \%$ accuracy in detecting damage in an in-service system and classifying them into respective locations and level of severities provided the suitable sensor point is selected for the scheme.

\section{Conclusion}

A damage detection scheme based on FRF is developed under ANN environment. The trained ANN network detected and classified the damages into respective locations and level of severities under both stationary and in-service conditions with high accuracy with the help of de-noising method used in ISMA. This happens due to three contribution factors. First, directly-measured FRF is one of the easiest dynamic responses to obtain in real-time, it is a more reliable and sensitive parameter to be used as input for damage detection process. Second, ISMA allows modal analysis to be performed during in-service condition, it gives static comparable FRF data through its de-noising method, i.e. Impact-Synchronous Time Averaging Technique. Third, the developed damage detection scheme using FRF is easy to use and well trained using the neural network pattern recognition toolbox and hence, able to detect and classify all the damages accurately.

\section{References}

1. Rahman, A.G.A., et al., Study of open crack in rotor shaft using changes in frequency response function phase. International Journal of Damage Mechanics, 2013. 22(6): p. 791-807.

2. Ong, Z.C., A.G.A. Rahman, and Z. Ismail, Determination of Damage Severity on Rotor Shaft Due to Crack Using Damage Index Derived from Experimental Modal Data. Experimental Techniques, 2014. 38(5): p. 18-30.

3. Ismail, Z. and Z.C. Ong, Honeycomb damage detection in a reinforced concrete beam using frequency mode shape regression. Measurement, 2012. 45(5): p. 950-959.

4. Ismail, Z., et al., Approach to Reduce the Limitations of Modal Identification in Damage Detection Using Limited Field Data for Nondestructive Structural Health Monitoring of a CableStayed Concrete Bridge. Journal of Bridge Engineering, 2012. 17(6): p. 867-875.

5. Ismail, Z., Application of residuals from regression of experimental mode shapes to locate multiple crack damage in a simply supported reinforced concrete beam. Measurement, 2012. 45(6): p. 1455-1461.

6. Hakim, S.J.S. and H.A. Razak, Modal parameters based structural damage detection using artificial neural networks - a review. Smart Structures and Systems, 2014. 14(2): p. 159-189.

7. Hakim, S.J.S., et al., Structural Damage Detection Using Soft Computing Method, in Structural Health Monitoring, Volume 5: Proceedings of the 32nd IMAC, A Conference and Exposition on Structural Dynamics, 2014, A. Wicks, Editor. 2014, Springer International Publishing: Cham. p. 143-151.

8. Hakim, S.J.S., H. Abdul Razak, and S.A. Ravanfar, Fault diagnosis on beam-like structures from modal parameters using artificial neural networks. Measurement, 2015. 76(Supplement C): p. 45-61.

9. Ong, Z.C. and C.C. Lee, Investigation of Impact Profile and Isolation Effect in Automated Impact Device Design and Control for Operational Modal Analysis. Journal of Dynamic Systems Measurement and Control-Transactions of the Asme, 2015. 137(9).

10. Cheet, L.H. and O.Z. Chao. Development of adaptive phase control impact device for enhancement of frequency response function in operational modal testing. in Proceedings of ISMA 2016 - International Conference on Noise and Vibration Engineering and USD2016 International Conference on Uncertainty in Structural Dynamics. 2016. 